\title{
Avaliação da relação entre parasitoses intestinais e fatores de risco para o HIV em pacientes com AIDS
}

\author{
Evaluation of the relatioship between of intestinal parasitoses \\ and risk factors for HIV infection in AIDS patients
}

\author{
Sérgio Cimerman, Benjamin Cimerman e David Salomão Lewi
}

\begin{abstract}
Resumo Estudo transversal em 200 pacientes portadores da síndrome da imunodeficiência adquirida (AIDS) na cidade de São Paulo, a fim de verificar se a presença de enteroparasitas está associada aos diversos fatores de risco para a infecção pelo HIV. Verificou-se que a diarréia foi mais freqüente no grupo que apresentou parasitose intestinal, sendo a diferença altamente significante $(p<0,0001)$. O parasita mais prevalente foi a Giardia lamblia em $32(16 \%)$ casos. A associacão entre a presença de parasitas nas fezes e os fatores de risco não revelou significado estatístico, principalmente para os patógenos Giardia lamblia e Cryptosporidium parvum $(p=0,99 ; p=0,69$, respectivamente). Devido à divergência na literatura, convém realizar estudos em outras áreas geográficas do país.
\end{abstract}

Palavras-chaves: Parasitas intestinais. Parasitologia. HIV. AIDS.

\begin{abstract}
We conducted a cohort survey on 200 AIDS patients in São Paulo, SP, Brazil, to verify if the presence of enteroparasites is associated with the various risk factors for HIV infection. Diarrhea was significantly more frequent in the group of patients presenting enteroparasitosis $(p<0.00001)$. Giardia lamblia, found in $32(16 \%)$ cases, was the most prevalent parasite. The presence of parasites in the stool samples was not significantly associated with risk factors for HIV infection, mainly when considering Giardia lamblia and Cryptosporidium parvum $(p=0.99$ and 0.69, respectively). The controversy found in the literature indicates that additional studies should be done in the different geographical regions of the country.
\end{abstract}

Key-words: Intestinal parasites. Parasitology. HIV. AIDS.

Com o advento da AIDS, protozoários que eram infreqüentes, assumiram grande relevância, como agentes de infecções oportunisticas, tais como criptosporidiose, isosporíase e microsporidiose 5 .

Os protozoários, especialmente Giardia lamblia e Entamoeba histolytica, são importantes causas de diarréia aguda em homens homossexuais, mesmo nos não-portadores de HIV2 912 18. Estudo comparativo, como o realizado em Toronto, mostrou que em 200 homens homossexuais e 100 heterossexuais, as freqüências de portadores de E. histolytica foram, respectivamente, de $27 \%$ e $1 \%$ e de G. lamblia nos mesmos respectivos grupos de $13 \%$ e $3 \%$. Outros estudos, como o de Mendes et al 13 , mostraram resultados semelhantes em homossexuais infectados pelo HIV ou não. Em São Paulo, em 771 amostras fecais de pacientes com AIDS, as taxas de amebíase e giardíase foram, respectivamente, de $5,2 \%$ e $8,5 \% 6$. Contudo, as formas clínicas invasivas da

Instituto de Infectologia Emílio Ribas, São Paulo, SP. Disciplina de Parasitologia da Universidade de Mogi das Cruzes, SP, Disciplina de Doenças Infecciosas e Parasitárias, Escola Paulista de Medicina, Universidade Federal de São Paulo, São Paulo, SP.

Endereço para correspondência: Dr. Sérgio Cimerman. Al. Jauaperi 890/111, 04523-014 São Paulo, SP.

Fax: $5511852-8744$

Recebido para publicação em 25/5/98. 
amebíase foram raramente descritas na literatura em pacientes com AIDS1 238.

Nos doentes com AIDS, a giardíase muitas vezes tem expressão clínica, e até exuberante, apesar do registro de prevalências semelhantes entre grupos populacionais de pacientes HIV positivos e negativos 13 .
Aliteratura, especialmente no Brasil, apresenta dados divergentes sobre a associação de parasitoses intestinais e a infecção pelo HIV. Daí, 0 interesse do presente trabalho, que avalia a prevalência dos enteroparasitas nos pacientes com AIDS, e a sua associação em relação ao comportamento sexual.

\section{CASUÍSTICA E MÉTODOS}

Foi realizado estudo transversal em 200 pacientes da Universidade Federal de São Paulo/Hospital São Paulo e do Instituto de Infectologia Emílio Ribas. Estes pacientes foram, previamente, informados que participariam de inquérito coprológico e epidemiológico e, estando de acordo, assinaram o termo de consentimento.

Os critérios de inclusão dos pacientes com infecção pelo HIV observaram as seguintes características: idade > 18 anos; uso ou não de terapia antiretroviral e/ou profilática; contagem de células $\mathrm{CD}_{4}<200$ células $/ \mathrm{mm}^{3}$, determinada por citometria de fluxo; soropositividade pelo teste ELISA para o HIV e/ou Western-blot.

O estudo foi conduzido de setembro de 1996 a abril de 1997. Uma amostra de fezes (10g) de cada paciente selecionado foi coletada em frasco de boca larga com tampa, contendo solução de formol a $10 \%(5 \mathrm{ml})$. Os frascos foram devidamente etiquetados com nome do paciente, data da coleta e número do registro do prontuário, e deixados à temperatura ambiente até o momento da realização do exame.

Os métodos coproparasitológicos utilizados foram o de Lutz10, também conhecido como Hoffman, Pons e Janer, Faust et al7 e Rugai, Mattos e Brisola19. E também, associadamente, as técnicas especiais de coloração, Kinyoun e Auramina-Rodamina (RG Kaminsky. Técnicas para laboratório de atención primaria de salud. Manual de Parasitologia. Organización Panamericana de la Salud, Honduras, 1966), para a pesquisa de oocistos dos protozoários Cryptosporidium parvum e Isospora belli.

$A$ variável quantitativa $C D_{4}$ não apresentou distribuição normal. Deste modo, foi utilizada a prova não paramétrica de Kruskal-Wallis na comparação dos valores de $C_{4}$ entre os diferentes grupos de parasitas. As variáveis qualitativas foram analisadas pelo teste do quiquadrado. O nível de significância de 0,05 $(\alpha=5 \%)$, correpondendo a probabilidade $(p)$ do erro tipo I foi considerada pequena e, portanto, com significado estatístico quando $<0,05(\alpha<5 \%)$.

\section{RESULTADOS}

Entre os 200 pacientes portadores de AIDS, havia 96 (48\%) heterossexuais, $17(18,5 \%)$ homossexuais, $10(5 \%)$ bissexuais, $24(12 \%)$ drogaditos, 7 (3,5\%) drogaditos e heterossexuais, $3(1,5 \%)$ drogaditos e homossexuais e $3(1,5 \%)$ drogaditos e bissexuais. Nove $(4,5 \%)$ casos relatavam exposição a transfusões de hemoderivados, $5(2,5 \%)$ eram hemofílicos e em $6(3 \%)$ casos, não se obteve informações precisas.
Quanto aos exames de fezes, 120 (60\%) não revelaram nenhum parasita intestinal e em $80(40 \%)$ o exame foi positivo. Os parasitas encontrados estão mencionados na Tabela 1. Chama a atenção, a ausência de E. histolytica. O poliparasitismo intestinal é mostrado na Tabela 2.

Como evidenciado na Tabela 3, a diarréia foi mais freqüente no grupo com parasitose intestinal

Tabela 1 - Parasitas intestinais em 200 pacientes portadores de AIDS provenientes de dois hospitais da cidade de São Paulo.

\begin{tabular}{lrr}
\hline Parasitas & \multicolumn{2}{c}{ Exame positivo } \\
\cline { 2 - 3 } Giardia lamblia & $\mathrm{n}^{0}$ & 32 \\
Entamoeba coli & 26 & 16,0 \\
Cryptosporidium parvum & 14 & 13,0 \\
Endolimax nana & 7 & 3,0 \\
Ascaris lumbricoides & 5 & 2,5 \\
Strongyloides stercoralis & 5 & 2,5 \\
Isospora belli & 4 & 2,0 \\
Blastocystis hominis & 1 & 0,5 \\
\hline
\end{tabular}


Revista da Sociedade Brasileira de Medicina Tropical 32:181-185, mar-abr, 1999.

Tabela 2 - Freqüência de infecções parasitárias múltiplas nos 200 pacientes com AIDS, provenientes de dois hospitais de São Paulo.

\begin{tabular}{|c|c|c|}
\hline Associações & $\mathrm{n}^{0}$ & $\%$ \\
\hline Giardia lamblia/Entamoeba coli & 3 & 1,5 \\
\hline Giardia lamblia/ Cryptosporidium parvum & 1 & 0,5 \\
\hline Giardia lamblia/Endolimax nana & 1 & 0,5 \\
\hline Giardia lamblia/Strongyloides stercoralis & 1 & 0,5 \\
\hline Giardia lamblia/Entamoeba coli/Cryptosporidium parvum/ & 1 & 0,5 \\
\hline Giardia lamblia/Entamoeba coli/Cryptosporidium parvum/Endolimax nana & 1 & 0,5 \\
\hline Isospora belli/Strongyloides stercoralis & 1 & 0,5 \\
\hline Entamoeba coli/Ascaris lumbricoides & 2 & 1,0 \\
\hline Total & 11 & 5,5 \\
\hline
\end{tabular}

Tabela 3 -Relação entre fezes diarréicas e presença de parasitas intetinais em 200 pacientes provenientes de dois hospitais da cidade de São Paulo.

\begin{tabular}{|c|c|c|c|c|c|}
\hline \multirow{3}{*}{ Fezes } & \multicolumn{4}{|c|}{ Parasitas } & \multirow{3}{*}{ Total } \\
\hline & \multicolumn{2}{|c|}{ resultado negativo } & \multicolumn{2}{|c|}{ resultado positivo } & \\
\hline & $\mathrm{n}^{0}$ & $\%$ & $\mathrm{n}^{0}$ & $\%$ & \\
\hline Diarréicas & 12 & 10,0 & 33 & 41,2 & 45 \\
\hline Não diarréicas & 108 & 90,0 & 47 & 58,8 & 155 \\
\hline Total & 120 & 100,0 & 80 & 100,0 & 200 \\
\hline
\end{tabular}

$\chi^{2}=25,12 p<0,0001$

$(41,2 \%)$, do que no grupo não-parasitado $(10 \%)$, sendo esta diferença altamente significativa $(p<0,0001)$. Não houve associação estatisticamente significativa entre os parasitas encontrados nas fezes e a contagem de células linfocíticas auxiliadoras (Tabela 4) e nem entre a situação de risco de se adquirir a infecção pelo HIV (Tabela 5).

Tabela 4- Comparação entre parasitas nas fezes e a contagem de células linfocíticas auxiliadoras $T+C D_{4}\left(c e l s / m^{3}\right)$ em 200 pacientes provenientes de dois hospitais da cidade de São Paulo.

\begin{tabular}{|c|c|c|c|c|c|}
\hline \multirow{2}{*}{$\begin{array}{l}\text { Parasitas } \\
\text { Resultado }\end{array}$} & \multicolumn{5}{|c|}{ Células CD4 } \\
\hline & média $\pm \mathrm{DP}$ & mediana & mínimo & máximo & $\mathrm{n}^{0}$ \\
\hline Negativo & $97,75 \pm 58,90$ & 97,5 & 3,0 & 199,0 & 120 \\
\hline Giardia lamblia & $78,41 \pm 70,66$ & 51,0 & 1,0 & 196,0 & 32 \\
\hline Entamoeba coli & $86,31 \pm 65,99$ & 87,5 & 1,0 & 199,0 & 26 \\
\hline Cryptosporidium parvum & $66,43 \pm 51,64$ & 55,5 & 13,0 & 182,0 & 14 \\
\hline Isospora belli & $59,50 \pm 59,24$ & 43,5 & 10,0 & 141,0 & 4 \\
\hline Endolimax nana & $118,14 \pm 70,94$ & 160,0 & 21,0 & 195,0 & 7 \\
\hline Strongyloides stercoralis & $68,40 \pm 71,23$ & 55,0 & 10,0 & 189,0 & 5 \\
\hline Ascaris lumbricoides & $98,60 \pm 88,64$ & 118,0 & 5,0 & 199,0 & 5 \\
\hline
\end{tabular}

$\mathrm{H}=2,93 \mathrm{p}=0,8179$

Demais parasitas encontrados no estudo apresentam freqüência insuficiente para o teste.

Tabela 5 - Comparação entre a presença de parasitas nas fezes e a categoria da exposição ao vírus HIV, em 200 pacientes provenientes de dois hospitais da cidade de São Paulo.

\begin{tabular}{|c|c|c|c|c|c|c|}
\hline \multirow[t]{2}{*}{$\begin{array}{l}\text { Parasitas } \\
\text { intestinais }\end{array}$} & \multicolumn{2}{|c|}{$\begin{array}{c}\text { Homossexuais } \\
\text { bissexuais }(n=53)\end{array}$} & \multicolumn{2}{|c|}{$\begin{array}{l}\text { Drogas } \\
(\mathrm{n}=37)\end{array}$} & \multicolumn{2}{|c|}{$\begin{array}{l}\text { Heterossexuais } \\
\qquad(n=103)\end{array}$} \\
\hline & $\mathrm{n}^{\circ}$ & $\%$ & $\mathrm{n}^{\circ}$ & $\%$ & $\mathrm{n}^{0}$ & $\%$ \\
\hline Negativo & 28 & 52,8 & 22 & 59,4 & 63 & 61,1 \\
\hline Giardia lamblia & 8 & 15,0 & 6 & 16,2 & 16 & 15,5 \\
\hline Entamoeba coli & 9 & 16,9 & 5 & 13,5 & 12 & 11,6 \\
\hline Cryptosporidium parvum & 5 & 9,4 & 3 & 8,1 & 6 & 5,8 \\
\hline Isospora belli & 1 & 1,8 & - & - & 3 & 2,9 \\
\hline Endolimax nana & 3 & 3,7 & - & - & 5 & 4,8 \\
\hline Strongyloides stercoralis & 1 & 1,8 & - & - & 4 & 3,8 \\
\hline Ascaris lumbricoides & 5 & 9,4 & 1 & 2,7 & - & - \\
\hline Blastocystis hominis & - & - & 1 & 2,7 & 1 & 0,9 \\
\hline
\end{tabular}

negativa: $\chi^{2}=1,02 \quad p=0,6013 ;$ G. lamblia: $\chi^{2}=0,02 p=0,9896 ;$ E. coli: $\chi^{2}=0,85 \quad p=0,6527 ;$ C. parvum: $\chi^{2}=0,73$ $p=0,6952$ 


\section{DISCUSSÃO}

Grande parte da morbidez na AIDS resulta de infecções oportunísticas do trato gastrointestinal.

As freqüências de parasitoses intestinais observadas no presente estudo, em geral, são semelhantes às encontradas na literatura. A freqüência de giardíase (16\%) observada é semelhante a descrita em outros trabalhos 6111314 , podendo apresentar alterações devido a variabilidade da área geográfica. A baixa freqüência de amebíase não-invasiva em pacientes com AIDS, também foi verificada no Recife (PE) por Magalhães et al $11(2,2 \%)$ e Alencar et al ${ }^{1}(1,3 \%)$.

A baixa prevalência de isosporíase (2\%) explica-se pelo fato dos pacientes estarem em uso de medicação profilática contra o Pneumocystis carinii15.

A diarréia esteve altamente associada com o encontro de enteroparasitas nos espécimes fecais, conforme, também, foi verificado por outros autores. Assim, Smith 20 verificou que em apenas $20 \%$ dos pacientes com AIDS, o quadro diarréico tinha etiologia obscura. Em mais de $50 \%$ dos casos, a diarréia estava associada à parasitose21.

A análise comparativa da contagem de células linfocíticas auxiliadoras $T^{+} C D_{4}$ e a presença de parasitas nas fezes não mostrou relação estatisticamente significante $(p=0,8179)$. O menor valor através da média ocorreu na presença de C. parvum $(66,4)$ e l. belli $(59,5)$ concordando com os dados de Moreno et al14 onde os valores de $\mathrm{CD}_{4}$ para criptosporidiose foram mais baixos do que para isosporíase.
O padrão de categoria de exposição ao HIV observado na população estudada segue a tendência epidemiológica documentada pela casuística de AIDS no Brasil, aonde o Ministério da Saúde aponta o contato sexual como principal forma de transmissão da doença que no período correspondente entre 1980 e 1997 foi responsável por $54,8 \%$ dos casos 4 . Estas evidências traduzem uma realidade mundial, com grande número de novas infecções adquiridas por via sexual e um substancial aumento de casos em mulheres 1622.

Muitas publicações referem elevada freqüência, em homens homossexuais, de patógenos como G. lamblia e E. histolytica ${ }^{18,}$ sendo que em relação a esta, pode chegar a $30 \% 17$. Nos pacientes com AIDS, estas taxas são bem menores. Em nossa amostra, não houve diferença estatística significante, em nenhuma categoria de exposição por intermédio de contaminação sexual, em relação aos parasitas G. lamblia, E. coli e C. parvum. As divergências encontradas na literatura, principalmente, em relação à $E$. histolytica e à patogenicidade das cepas isoladas, justificam a realização de mais estudos em outras áreas geográficas.

Concluindo, a freqüência baixa ou nula de amebíase em pacientes com AIDS merece estudos adicionais, não só clínico-epidemiológicos mas, também, investigações no campo acerca da patogenicidade das cepas de $E$. histolytica isoladas. A freqüência de giardíase, provavelmente, decorre da elevada distribuição da mesma, na população em geral. Contudo, em pacientes com AIDS e quadro diarréico, a investigação parasitológica deve ser rotineira em nosso meio.

\section{REFERÊNCIAS BIBLIOGRÁFICAS}

1. Alencar LCA, Magalhães V, Melo VM, Akai I, Magalhães M, Kobayashi S. Ausência de amebíase invasiva em aidéticos homossexuais masculinos, no Recife. Revista da Sociedade Brasileira de Medicina Tropical 29: 319-322, 1996.

2. Allason-Jones E, Mindell A, Sargeaunt P, Williams P. Entamoeba histolytica as a comensal intestinal parasite in homosexual men. New England Journal of Medicine 315: 353-356, 1986.

3. Blanshard C, Collins C, Francis N, Gazzard BG. Invasive amoebic colitis in AIDS patients. AIDS 6:1043-1044, 1992.

4. Brasil MS. Programa Nacional de DST/AIDS. Associação Brasileira Interdisciplinar de AIDS (ABIA). Boletim Epidemiológico (AIDS) 10: 1-50, 1997.

5. Cimerman S, Cimerman B, Lewi DS. Parasitoses intestinais. Visão crítica de sua importância em nossso meio. Ars Curandi 31: 5-9, 1998.
6. Dias RMDS, Pinto WP, Chieffi PP, Magini ACS, Torres DNMAGV, Del Bianco R, Ferrari L. Enteroparasitoses em pacientes acometidos pela síndrome de imunodeficiência adquirida (AIDS/SIDA). Revista Adolfo Lutz 48: 63-67, 1988.

7. Faust EC, Russell PF, Jung RC. Craig and Faust's Clinical Parasitology. 8th edition, La Febiger, Philadelphia, 1970.

8. Jessurun J, Barrón-Rodriguez LP, Fernández-Ávila M. The prevalence of invasive amebiasis is not increased in patients with AIDS. AIDS 6: 307-309, 1992.

9. Keystone JS, Keystone DL, Proctor EM. Intestinal parasitic infections in homosexual men: prevalence, symptoms and factors in transmission. Canadian Medical Association Journal 123: 512-514, 1980.

10. Lutz A. O Schistosomum mansoni e a schistosomatose segundo observações feitas no Brazil. Memórias do Instituto Oswaldo Cruz 11: 121-155, 1919. 
11. Magalhães V, Lima RA, Melo VM, Magalhães M, Alencar LCA, Carrera M, Lucena TALM, Rangel F. Enteroparasitoses em pacientes infectados pelo vírus da imunodeficiência humana (HIV) no Recife. Revista Brasileira de Medicina 50: 439-445, 1993.

12. Markell EK, Havens RF, Kuritsubo RA, Wingerd J. Intestinal protozoa in homosexual men of the São Francisco Bay area: prevalence and correlates of infection. American Journal of Tropical Medicine and Hygiene 32: 239-245, 1984.

13. Mendes OC, Dzmulewicz G, Menghi C, Torres S, Gonzales G, Gatta C. Comparación de indices de infestaciones por enteroparasitos entre problaciones HIV positivos y negativos. Medicina (Buenos Aires) 54: 307-310, 1994.

14. Moreno A, Gatell JM, Mensa J, Valls ME, Vila J, Claramonte X, Miró JM, Mallolas J, Zamora L, Lozano L, Trilla A, Storiano E. Incidencia de enteropatógenos en pacientes con infección por el virus de la imunodeficiência humana. Medicina Clinica (Barcelona) 102: 205-208, 1993.

15. Pape JW, Verdier RI, Johnson Jr WD. Treatment and prophilaxis of Isospora belli infection in patients with the acquired immunodeficiency syndrome. New England Journal of Medicine 320: 1044-1047, 1989.
16. Quinn TC. Global burden of the HIV pandemic. Lancet 348:99-106, 1996.

17. Reed SL, Wessel DW, Davis CF. Entamoeba histolytica infection and AIDS. American Journal of Medicine 90: 269-271, 1991.

18. Rojas PN, Queroles HM. Infecciones en el homosexual. Gen 39: 195-202, 1985.

19. Rugai E, Mattos T, Brisola AP. Nova técnica para isolar larvas de nematóides das fezes: modificações do método de Baermann. Revista do Instituto Adolfo Lutz 14: 5-8, 1954.

20. Smith PD. Diarréia infecciosa nos pacientes com AIDS. Clínicas Gastroenterológicas da América do Norte 3: 369-384, 1993.

21. Sturgess I, Greenfield SM, Teare J, O'Doherty MJ. Ulcerative colitis developing after amoebic dysentery in a haemophiliac patient with AIDS. Gut 33 408-410, 1992.

22. Tatantola DJM, Mann JM. Global expansion of HIV infection and AIDS. Hospital Practice 31: 63-79, 1996.

23. World Health Organization. Informal consultation on intestinal protozoal. World Health Organization/ Panamerican Health Organization. Mexico, 1991. 